\title{
LABORATÓRIO DE NUTRIÇÃo E METABOLISMO APLICADO À ATIVIDADE MOTORA
}

\author{
Antonio Herbert LANCHA JUNIOR*
}

\section{INTRODUÇÃO}

A nutrição aplicada a atividade motora teve seu primeiro congresso internacional e foi identificada como área do conhecimento em 1990, em congresso realizado pela Nutrition Society, na Grécia.

Nesse evento, recebeu destaque a apresentação de Singh (1992) que definiu como população a ser investigada pela nutrição aplicada a atividade motora dois grupos específicos: os individuos comuns, praticantes de atividade física regular que apresentam o objetivo de saúde e/ou estético; e os indivíduos que objetivam 0 desempenho, conceitualmente definidos como atletas.

Recentemente, Lancha Junior (1999) diferenciou essa população em três grupos, sendo: os indivíduos comuns, os atletas (igualmente aos descritos por Singh) e além desses os portadores de doenças, que teriam uma abordagem distinta dos outros dois.

Dessa forma, a investigação científica em nutrição aplicada a atividade motora acaba permeando esses três grupos, porém sempre com uma concentração maior em um.

Historicamente, as pesquisas envolvendo nutrição e atividade motora no Brasil, tiveram início na Escola de Educação Física e Esporte da USP (EEFEUSP) muito antes da caracterização da área e da existência de laboratório. $\mathrm{O}$ responsável por isso foi um dos pioneiros nessa área de investigação, o Prof.Dr. Sérgio Miguel Zucas. A atuação do professor Zucas foi determinante na formação de diversos pesquisadores que teriam, posteriormente, envolvimento com ensino na EEFEUSP. Dentre eles podemos destacar o Prof.Dr. Rubens Lombardi Rodrigues e Prof.Dr. Eduardo Kokubun, além de outros com atuação de destaque em diversos centros universitários brasileiros.

O Laboratório de Nutrição, assim como todos os outros, surgiu em meados de 1988, quando o espaço físico destinado aos laboratórios foi dividido entre as áreas estabelecidas na EEFEUSP e implementado ao longo dos anos de existência.

Hoje o Laboratório de Nutrição conta com os docentes Prof.Dr. Benedito Pereira e Prof.Dr. Antonio Herbert Lancha Junior (coordenador), e recursos financeiros da FAPESP para equipamentos, materiais de consumo e bolsas de estudo. Possui, com isso, duas linhas distintas de investigação: metabolismo de aminoácidos e atividade motora sob responsabilidade do professor Lancha Junior e metabolismo do oxigênio na atividade motora sob responsabilidade do professor Pereira (em fase de implantação).

Essas duas linhas de investigação permitem diversas interfaces com a área metabólica, de onde surgem os estudos publicados pelo Laboratório de Nutrição.

O espaço físico do Laboratório é composto por duas áreas distintas sendo uma destinada aos estudos com animais, localizado na EEFEUSP, e outra localizada no Centro de Práticas Esportivas da USP (CEPEUSP). Essa segunda área no CEPEUSP foi construída com apoio financeiro da FAPESP, em 1997, e homologada pela Congregação da EEFEUSP no mesmo ano.

Atualmente, os estudos desenvolvidos em nosso Laboratório compreendem a relação entre 0 metabolismo dos aminoácidos com desenvolvimento de resistência periférica a insulina, além da sua relação com a tolerância ao esforço.

Coordenador do Laboratório de Nutrição e Metabolismo aplicado à Atividade Motora da Escola de Educação Física e Esporte da Universidade de São Paulo. 


\section{PROJETOS EM DESENVOLVIMENTO}

\section{Estudos relacionando atividade física, nutrição e obesidade}

A obesidade já é considerada uma epidemia mundial, não respeitando o estado de desenvolvimento dos países. Com isso, diversos estudos envolvendo atividade física e controle nutricional são desenvolvidos, gerando contradições a respeito da abordagem a ser dada em tal situação (Pereira, Francischi, Klopfer, Sawada, Santos, Vieira, Campos \& Lancha Junior, 1999). A atividade física favorece a perda de gordura corporal na medida em que determina balanço energético negativo sem necessariamente impor grande restrição alimentar. Dados obtidos em nosso Laboratório demonstram que a população obesa brasileira apresenta grande consumo de gordura e proteína na dieta, com restrição ao consumo de carboidratos (Francischi, Klopfer, Pereira, Campos, Sawada, Santos, Vieira \& Lancha Junior, 1999). Além disso, observamos que a obesidade, no Brasil, ocorre nas mulheres estudadas em nosso Laboratório por volta dos 19 anos de idade, 0 que evidencia a pouca possibilidade da mesma ser de origem genética (Freitas, Francischi, Pereira, Vieira, Klopfer, Santos, Sawada, Campos \& Lancha Junior, 1999). Esses fatos evidenciam a importância da atividade física e da reeducação nutricional para prevenir as conseqüências da obesidade mantida, como a resistência periférica, diabetes tipo 2, hipercolesterolemia, etc.

A pergunta que surge no tocante a atividade destinada à população obesa gira em torno da atividade de resistência, como a caminhada, que garante grande utilização de lipídeos como fonte de energia, auxiliando a redução da adiposidade no organismo. Já a atividade contra-resistência, na qual o trabalho com sobrecarga favorece o aumento da massa muscular, poderia garantir maior gasto energético pelo aumento da massa magra e, conseqüentemente, determinar maior gasto calórico.

Com o intuito de avaliar qual intervenção seria mais indicada para esse fim, desenvolvemos um estudo comparando essas intervenções (Francischi et alii, 1999). De modo oposto ao que idealizamos, a atividade mais efetiva na redução da gordura corporal foi a mantida ao redor de $60 \%$ do consumo máximo de oxigênio por três dias na semana, por quatro semanas.
Outro dado bastante importante obtido em nosso Laboratório foi que sem 0 controle nutricional, a população obesa tende a realizar aumento do consumo de calorias de forma compensatória. Assim, o possível efeito redutor da adiposidade imposto pela atividade física somente é perceptível com a intervenção nutricional.

\section{Estudos relacionando metabolismo de aminoácidos e resistência periférica a insulina}

Como descrito acima, a população obesa apresenta como padrão alimentar maior ingestão de lipídeos na dieta com comprometimento da ingestão de carboidratos. Esse fato foi decorrente de uma cultura estabelecida de que seriam os alimentos fonte de carboidratos os responsáveis pela determinação da gordura corporal. De fato, os alimentos que apresentam grande concentração de carboidratos determinam elevação da concentração de glicose e, conseqüentemente, de insulina. Essa, por sua vez, atua no adipócito como inibidora da lipólise. É evidente que isso interfere na concentração de gordura corporal, mas está longe de ser 0 responsável pela adiposidade excessiva apresentada pelo obeso.

A baixa ingestão de carboidratos na dieta do obeso impõe ao organismo algumas adaptações no sentido de preservar a manutenção da glicemia para regiões de consumo prioritário desse nutriente, como as células do sistema nervoso central.

O substrato energético mais susceptível a conversão à glicose são os aminoácidos, que podem ser oriundos da ingestão alimentar ou do catabolismo do organismo.

Recentemente, verificamos que a ingestão de aminoácidos suplementados na dieta de ratos (aspartato e asparagina $45 \mathrm{mg}$ cada) determina quadro de resistência periférica à ação da insulina na captação de glicose (RPICG), alterando a secreção de insulina e reduzindo as concentrações de glicogênio muscular (Costa, Sawada, Marquezi \& Lancha Junior, 1999; Lancha Junior, 1996, 1997a; Lancha Junior, Han, Hansen \& Hollosky, 1995; Sawada, Costa, Marquezi \& Lancha Junior, 1999).

O modelo responsável pela RPICG, imposto pela suplementação de aminoácidos, coincide com o proposto em 1991 por Marshall. Segundo Traxinger \& Marshall (1989), a RPICG pode ser induzida no músculo pelo aumento da disponibilidade de glutamina (aminoácido produzido pelo metabolismo aeróbio muscular) e 
glicose. Esses nutrientes seriam, então, convertidos a glicosamina (metabólito oriundo da condensação de frutose com um grupamento amínico da glutamina) que induziria menor sinalização intracelular da ação da insulina, resultando na RPICG.

Dessa forma, o obeso, ao comprometer a ingestão alimentar de fontes de carboidratos, favoreceria maior processamento de aminoácidos. Isso por sua vez, levaria a maior síntese de glicosamina e a instalação da RPICG.

\section{Estudos relacionando suplementação de aminoácidos e ultra-estrutura celular e desempenho}

Como descrito acima, a suplementação de aminoácidos promove alteração da capacidade de captação de glicose, modulando o metabolismo dos carboidratos. A interface entre metabolismo de aminoácidos e os outros nutrientes é muito grande, porém poucas investigações científicas ocorreram nesse sentido.

Lancha Junior et alii (1995) demonstraram que a suplementação de aspartato, asparagina e carnitina (45 mg, $45 \mathrm{mg}$ e $90 \mathrm{mg}$, respectivamente) promove maior tempo de tolerância ao esforço em ratos submetidos a natação.

Os estudos realizados com esses suplementos obrigatoriamente passam pelo modelo experimental (experimental, no texto, representa estudo com animais, no caso, ratos albinos Wistar) anteriormente à sua avaliação em humanos, pois sendo grande parte dos nutrientes substâncias bioativas, ou seja, que produzem respostas biológicas (bioquímicas, celulares, fisiológicas) não devem ser inicialmente usadas em humanos devido ao comprometimento funcional, além das limitações de investigação celular.

A confirmação das afirmações acima foi obtida nos trabalhos de Lancha Junior, Santos, Palanch \& Curi (1997b), que encontraram alteração da ultra-estrutura celular muscular de ratos suplementados com os mesmos aminoácidos acima citados, na mesma dosagem. Das principais alterações destacam-se as modificações mitocondriais (tamanho e forma), e a destruição da linha Z.

Mantendo, então, o modelo experimental na investigação do desempenho, recentemente, Marquezi, Costa, Sawada \& Lancha Junior (1999) demonstraram que os aminoácidos aspartato e asparagina podem promover maior fluxo de NADH do sarcoplasma para 0 interior mitocondrial via sistema de lançadeira aspartato-malato. Esse sistema possui a capacidade de transferir elétrons para a mitocondria sem promover aumento nas concentrações de lactato.

Como conseqüência dessa menor concentração de lactato, os animais suplementados apresentaram maior tempo de resistência ao esforço em atividade intensa (supra-limiar metabólico).

Assim, a suplementação de aminoácidos parece modular a capacidade de tolerância ao esforço moderado e intenso, porém apresenta conseqüências como as modificações ultraestruturais e indução do quadro de RPICG.

\section{ESTUDOS FUTUROS}

No presente momento, o Laboratório de Nutrição desenvolve estudos que têm por objetivo determinar:

$>\mathrm{O}$ tipo mais efetivo de atividade física na redução da adiposidade e qual a região (intraabdominal ou subcutânea) mais afetada com e sem a intervenção nutricional. Esse estudo conta com o apoio da FAPESP (bolsa de Iniciação Científica) e do Hospital Nove de Julho, onde são realizadas as tomografias computadorizadas.

$>$ Efeito da suplementação de creatina sobre a concentração de lactato plasmático em ratos submetidos a natação. Esse estudo está em julgamento na FAPESP (bolsa de Mestrado), com resultados preliminares já obtidos.

$>$ Efeito da freqüência alimentar e da composição de macro-nutrientes no desenvolvimento da obesidade em ratas (albino Wistar). Esse projeto está em julgamento na FAPESP (bolsa Mestrado/UNICAMP), com início programado para março de 2000.

$>$ Efeito da dieta rica em lipídeos no desenvolvimento de obesidade em ratas e possivel efeito sobre a ultra-estrutura muscular. $O$ projeto conta com 0 apoio FAPESP (bolsa Mestrado/UNICAMP) e está em andamento, já com os primeiros dados obtidos, restando ainda a avaliação da resistência periférica a insulina e da ultraestrutura celular. 
$>$ Suplementação de aminoácidos aspartato e asparagina associado ao treinamento moderado na RPICG. Esse estudo conta com o apoio da CAPES (bolsa de Mestrado) e está em desenvolvimento com ${ }^{\circ}$ alguns experimentos já realizados.

$>\quad$ Suplementação de aminoácidos glutamina na RPICG. Esse estudo conta com o apoio da CAPES (bolsa de Mestrado) e está em desenvolvimento com alguns experimentos já realizados.

$>$ Suplementação de aspartato e asparagina sobre as concentrações de lactato plasmático em ratos. Trabalho a ser apresentado para obtenção do título de Mestre.

O Laboratório de Nutrição conta hoje com recursos gerados pelo Programa de Atividade Física e Controle Nutricional para Mulheres Obesas de 20 a 40 anos e recursos oriundos de projetos junto à FAPESP Além das pesquisas relatadas, outros estudos estão em fase de elaboração para apresentação à FAPESP

\section{REFERÊNCIAS BIBLIOGRÁFICAS}

COSTA, A.S.; SAWADA, L.A.; MARQUEZI, M.L.; LANCHA JUNIOR, A.H. Exercício físico, suplementação nutricional de aminoácidos e captação de glicose. Revista Brasileira de Nutrição Clínica, v.14, p.40-50, 1999.

FRANCISCHI, R.P.; KLOPFER, M.; PEREIRA, L.O; CAMPOS, P.L.; SAWADA, L.A.; SANTOS, R.; VIEIRA, P.; LANCHA JUNIOR, A.H. Efeito da intensidade da atividade fisica e da dieta hipocalórica sobre o consumo alimentar, a composição corporal e a colesterolemia em mulheres obesas. Revista Brasileira de Nutrição Clínica, v. 14, p. 1-8, 1999.

FREITAS, C.S.; FRANCISCHI, R.P.; PEREIRA, L.O.; VIEIRA, P.; KLOPFER, M.; SANTOS, R.; SAWADA, L.A.; CAMPOS, P.L.; LANCHA JUNIOR, A.H. Behavioral aspects of obesity in Brazilian women. 1999. (Submetido à publicação no The British Joumal of Nutrition)

LANCHA JUNIOR, A.H. Atividade fisica, suplementação nutricional de aminoácidos e resistência periférica à insulina. Revista Paulista de Educação Física, v.10, n.1, p.68-75, 1996.
Efeito da suplementaçāo de aminoácidos (aspartato e asparagina) sobre 0 transporte de glicose em músculo esquelético de ratos. São Paulo, 1997a. 90p. Tese (Livre-Docência) - Escola de Educação Física e Esporte, Universidade de São Paulo.

Nutrição aplicada às atividades fisica $e$ esportiva. In: GHORAYEB, N.; BARROS NETO, T.L., eds. $O$ exercício: preparação fisiológica, avaliação médica, aspectos especiais e preventivos. São Paulo, Atheneu, 1999. Cap.8, p.75-85.

LANCHA JUNIOR, A.H.; HAN, D.H.; HANSEN, P.A.; HOLLOSZY, J.O. Effect of aspartate and asparagine supplementation in the glucose transport activity in epitrochlearis muscle. Medicine and Science in Sports and Exercise, v.27, p.S146, 1995. Supplement.

LANCHA JUNIOR, A.H.; SANTOS, M.F; PALANCH, A.C.; CURI, R. Supplementation of aspartate and asparagine and carnitine in the diet causes marked changes in the ultrastructure of soleus muscle. Journal of Submicroscopic Cytology and Pathology, v.29, n.3, p.405-8, 1997 b.

MARQUEZI, M.L.; COSTA, A.S.; SAWADA, L.A.; LANCHA JUNIOR, A.H. Possível efeito da suplementação aguda dos aminoácidos aspartato e asparagina sobre o metabolismo de lactato e sua possível implicação sobre 0 limiar anaeróbio. Revista Brasileira de Nutrição Clínica, v.14, p.519, 1999.

PEREIRA, L.O.; FRANCISCHI, R.P.; KLOPFER, M.; SAWADA, L.A.; SANTOS, R.; VIEIRA, P.; CAMPOS, P.L.; LANCHA JUNIOR, A.H. Obesidade e suas implicações: ação da atividade fisica e controle nutricional. Revista Brasileira de Nutrição Clínica, v.14, p.9-17, 1999.

SAWADA, L.A.; COSTA, A.S.; MARQUEZI, M.L.; LANCHA JUNIOR, A.H. Suplementação de aminoácidos e resistência à insulina. Revista Brasileira de Nutrição Clínica, v.14, p.31-9, 1999.

SINGH, V.N. A current perspective on nutrition and exercise. Journal of Nutrition, v.122, p.760-5, 1992.

TRAXINGER, R.R.; MARSHALL, S. Role of amino acids in modulating glucose-induced desensitization of the glucose transport system. Journal of Biological Chemistry, v.264, p.20910-6, 1989.

ENDEREÇO: Antonio Herbert Lancha Junior

EEFEUSP

Av. Prof. Mello Moraes, 65

05508-900 - São Paulo - SP - BRASIL 